\title{
THE STRUCTURE AND SYSTEM OF MOTIVATIONS OF THE TEACHING STAFF PROFESSIONAL ACTIVITIES
}

\author{
NANA LUKHUTASHVILI \\ Assistant Professor of Akaki Tsereteli State University \\ Associate Professor of Kutaisi University, Georgia \\ nana.lukhutashvili@atsu.edu.ge \\ nana.lukhutashvili@unik.edu.ge
}

\section{TEA VALISHVILI}

Associate Professor of Akaki Tsereteli State University

Associate Professor of Kutaisi University, Georgia

tea.valishvili@atsu.edu.ge

tea.valishvili@unik.edu.ge

Abstract. In order to ensure that the higher education institutions attain the desired objectives, it is very important to motivate employees to work effectively, which directly depends on their work motivation.

When managing motivation, the manager's job is to activate the motivations that already lie in the employees.

Specific activities of the teaching staff in the higher education system are conditioned by motivations of work activities, as well as by the joint action of motivations for choosing profession and motivations for choosing workplace, the ratio of which guides the conduct of employees and creates its motivational structure.

Virtually every worker has his/her own motivational structure, in which the leading place is occupied by the motivating core, which is the set of leading motives and stimuli that guide workers at a given point in time.

In order to reveal the motivating core in the structure of the teaching staff, we conducted a survey at Akaki Tsereteli State University. A total of 252 respondents took part in the survey of the teaching dtaff. As a result of the survey, motivations that are mostly related to the content of labor and the specific labor conditions typical for the higher education institutions.

In order to succeed in organizations and their structure, it is not only desirable, but essential to create, maintain and operate a staff motivation system in continuous development mode.

Based on our research, we have identified the areas of motivation and stimulation that are most relevant for Akaki Tsereteli State University today, and based on them, we have developed the motivational program that, in our opinion, will help the university to achieve its strategic goals and successfully meet the challenge.

KEYWORDS: MOTIVATION, LEADING MOTIVES, MOTIVATIONAL STRUCTURE, MOTIVATIONAL

CORE, MOTIVATIONAL PROGRAM.

For citation: Lukhutashvili N., Valishvili T. (2021). The structure and system of motivations of the teaching staff professional activities. Globalization and Business, №11, 112- 117. (In Georgian). https://doi.org/10.35945/gb.2021.11.016 


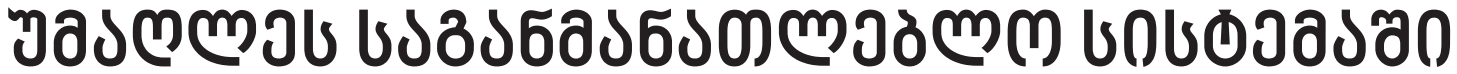

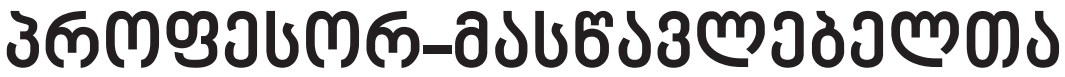

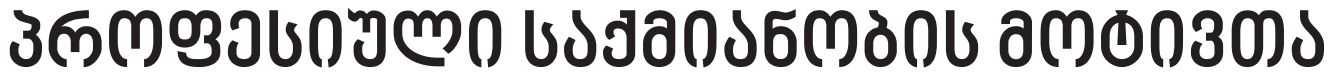

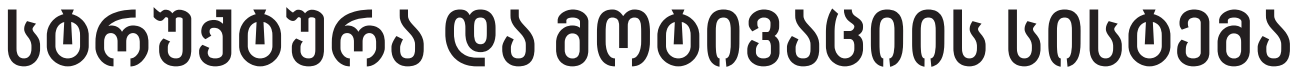

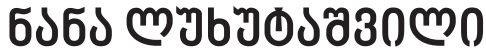

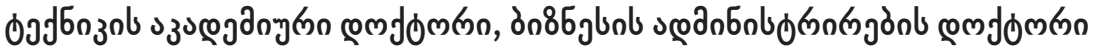

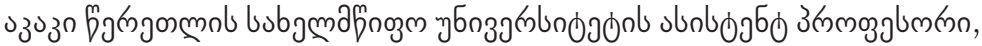

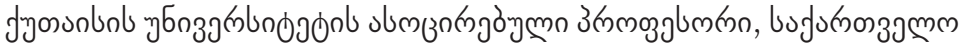

nana.lukhutashvili@atsu.edu.ge

nana.lukhutashvili@unik.edu.ge

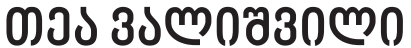

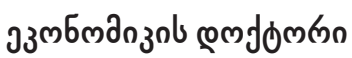

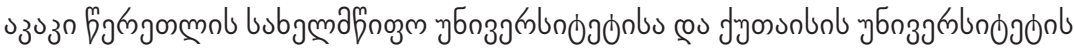

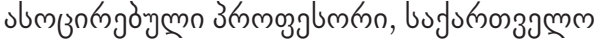

tea.valishvili@atsu.edu.ge

tea.valishvili@unik.edu.ge

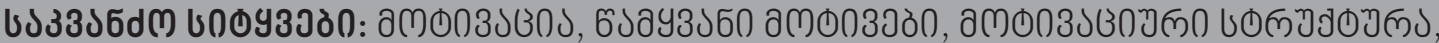

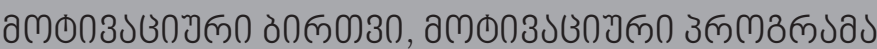

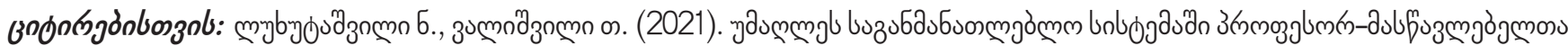

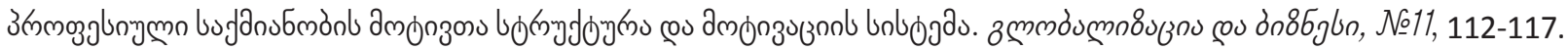

https://doi.org/10.35945/gb.2020.11.016

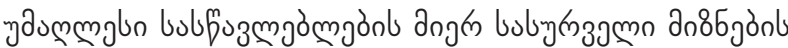

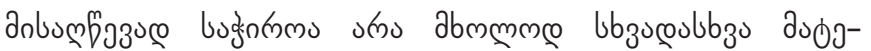

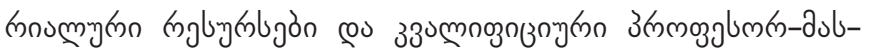

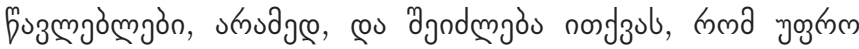

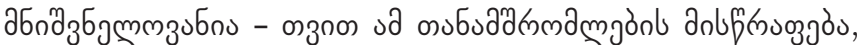

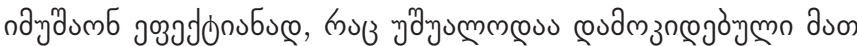

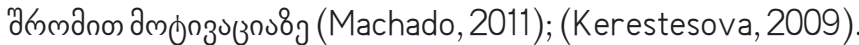

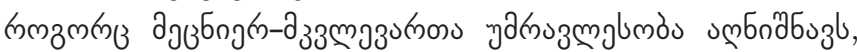

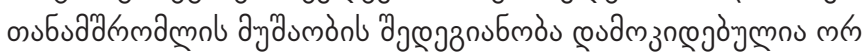

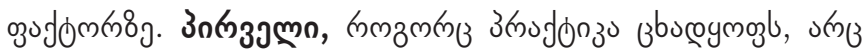

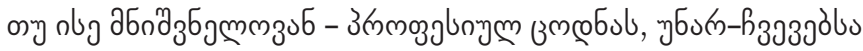

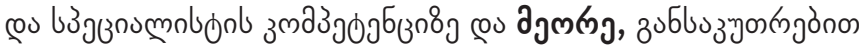

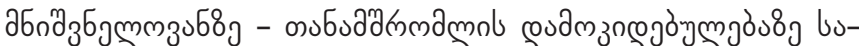

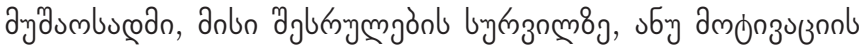
сомб

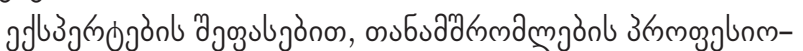

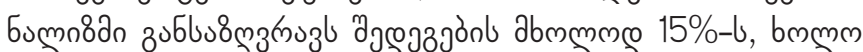

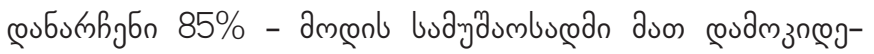

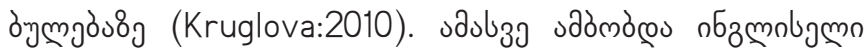

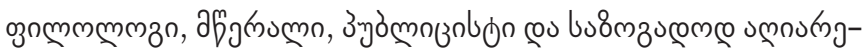

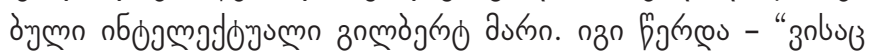

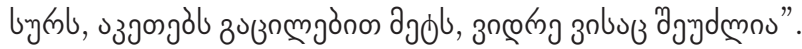

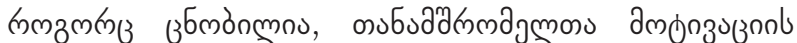

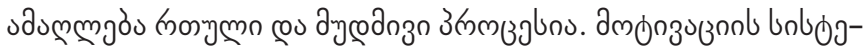

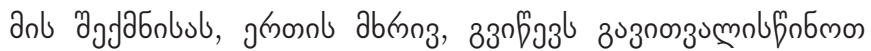

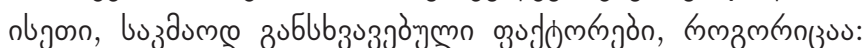

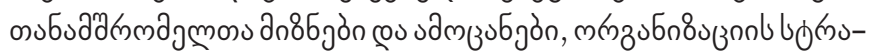

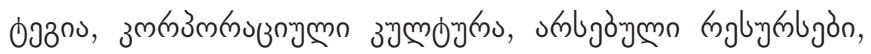

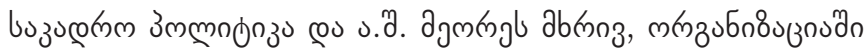

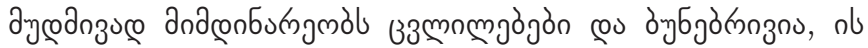

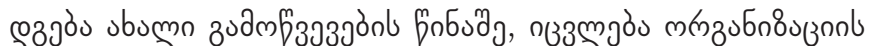

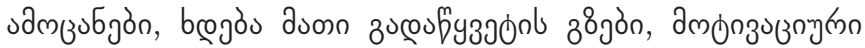

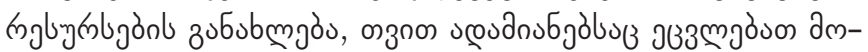

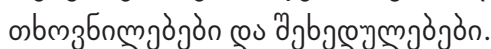

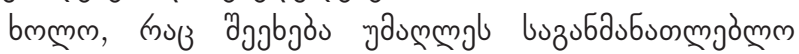

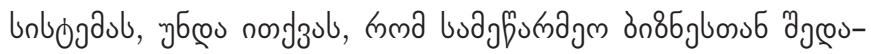




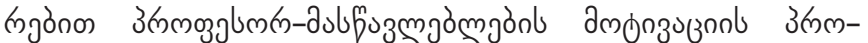

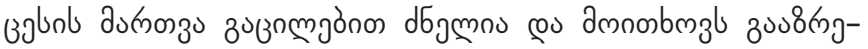

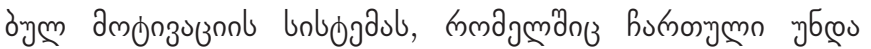

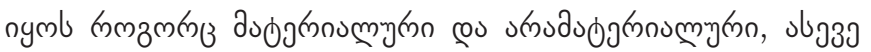
उmonon

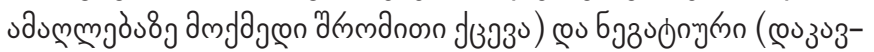
चn r.

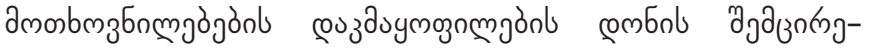

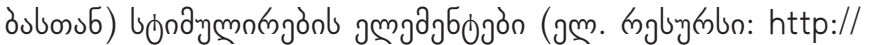
motivtruda.ru/ pozitivnoe-i-negativnoe.htm).

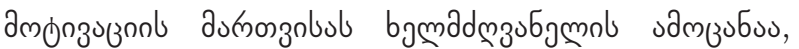

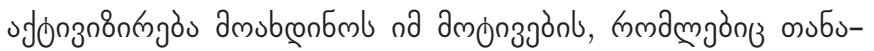

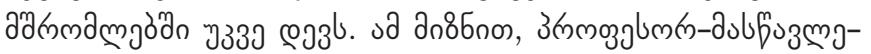

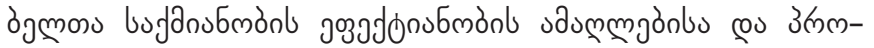

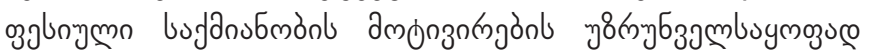

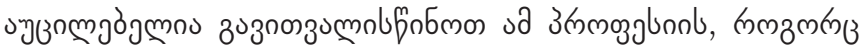

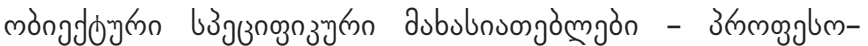

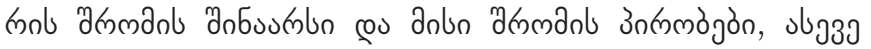

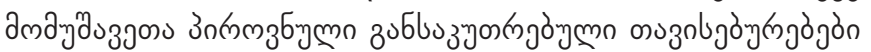

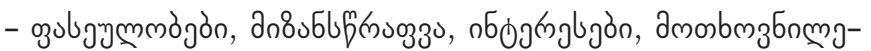

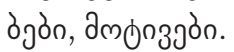

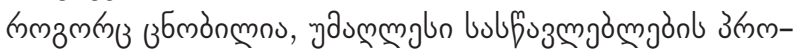

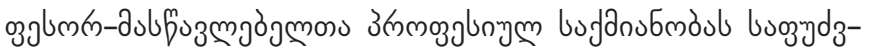

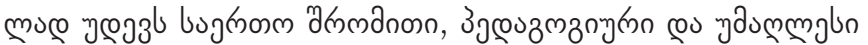

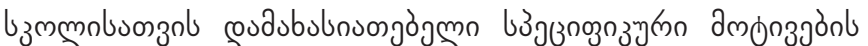

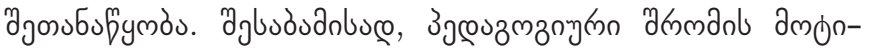

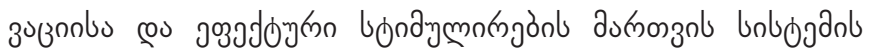

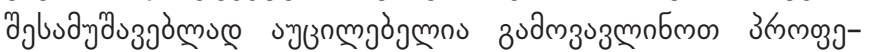

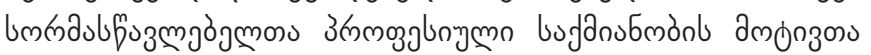

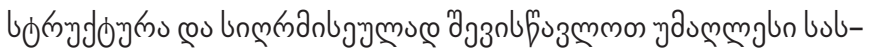

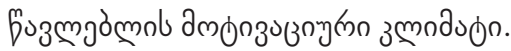

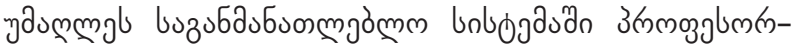

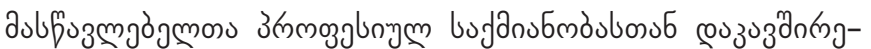

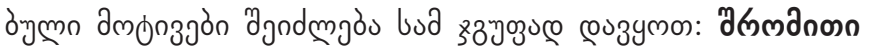

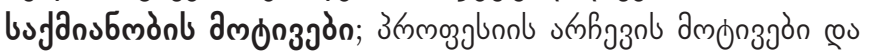

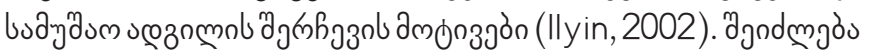

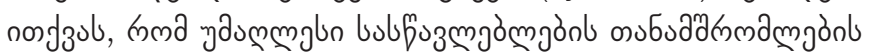

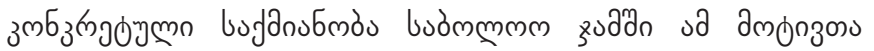

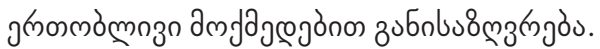

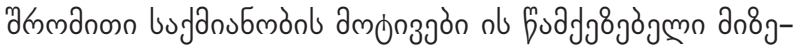

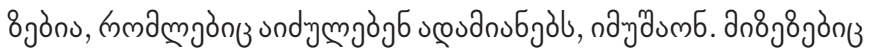

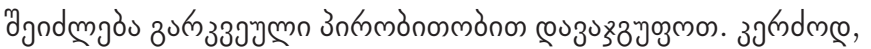

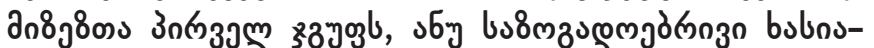

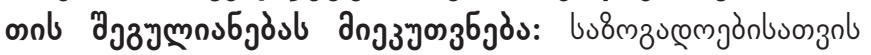

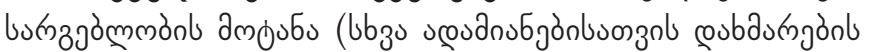

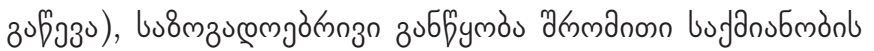

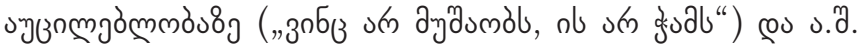

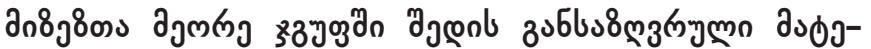

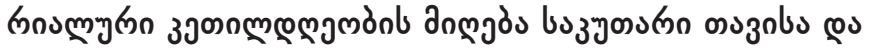

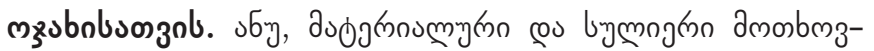

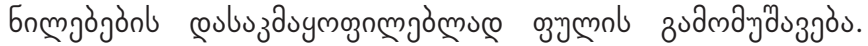

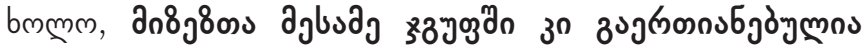

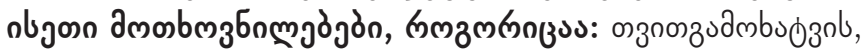

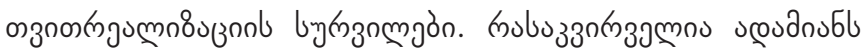

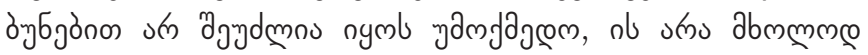

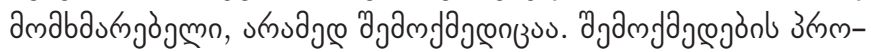

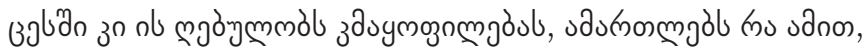

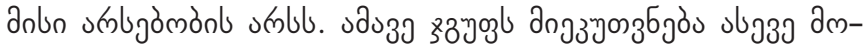

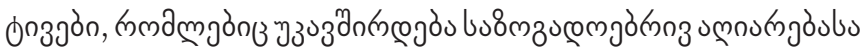

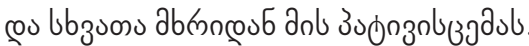

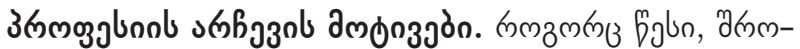

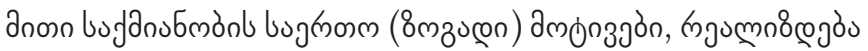

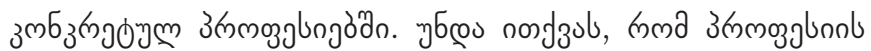

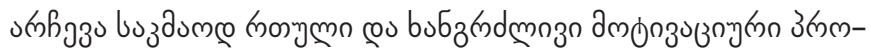

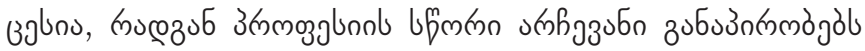

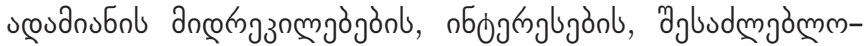

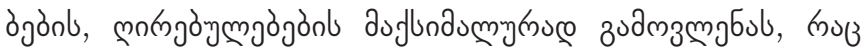

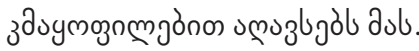

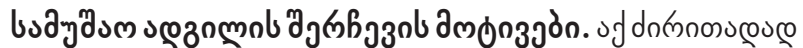

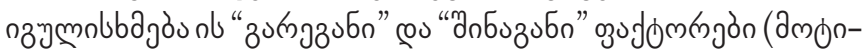

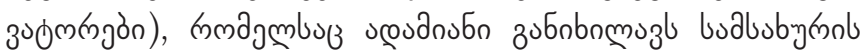
u ک

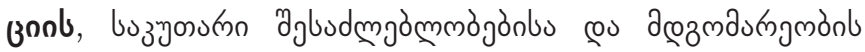

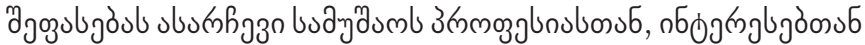

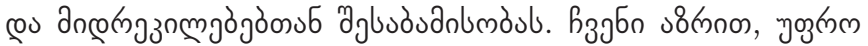

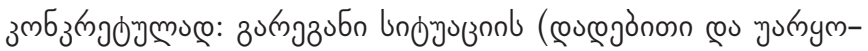

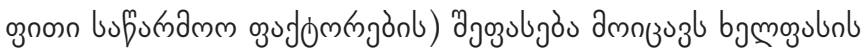

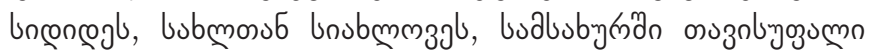

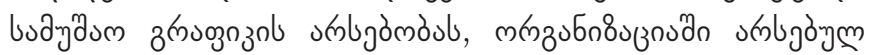

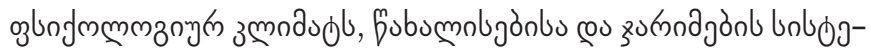

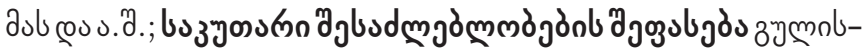

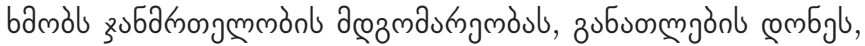

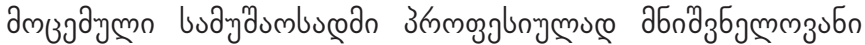

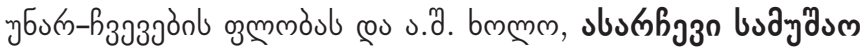

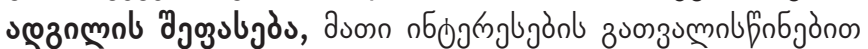

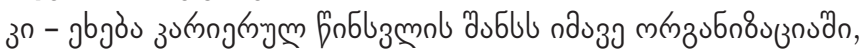

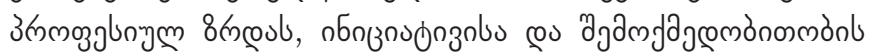

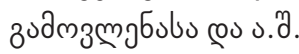

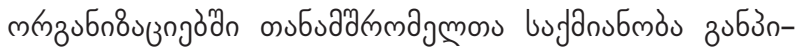

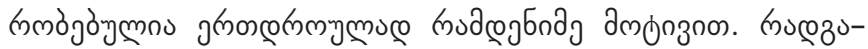

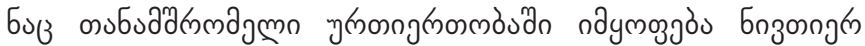

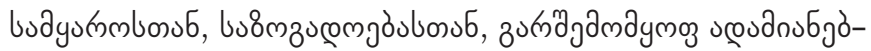

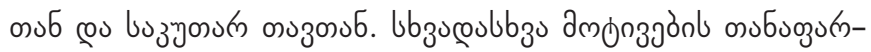

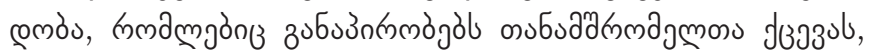

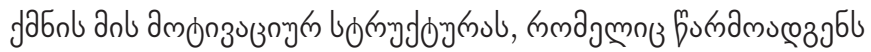

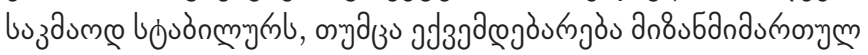

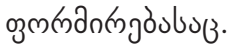

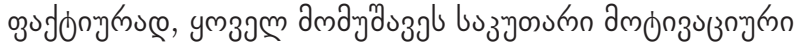

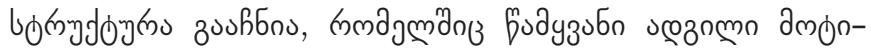

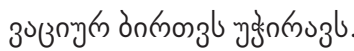




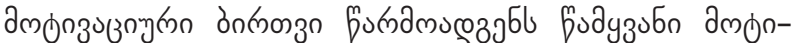

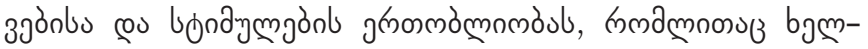

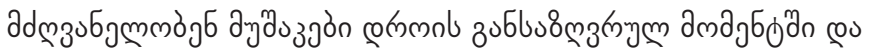
zublus

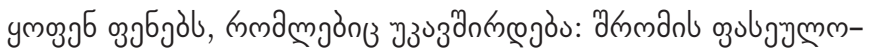

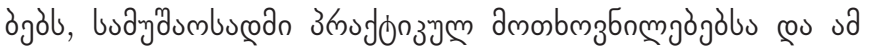

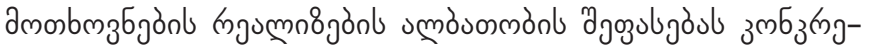
oym lnoyoznodn.

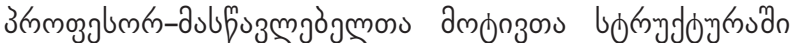

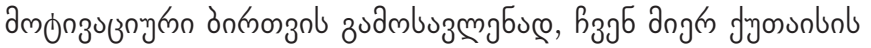

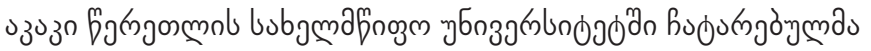

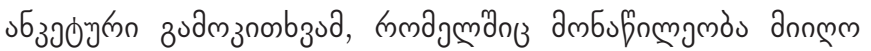

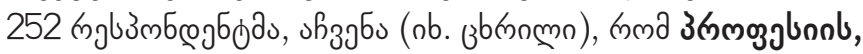

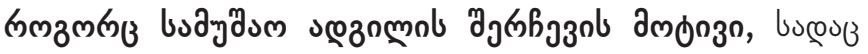

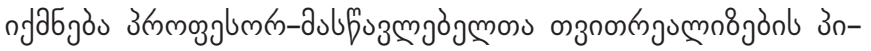

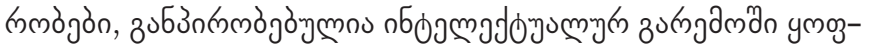

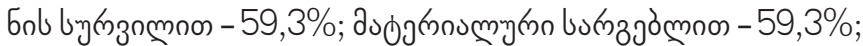

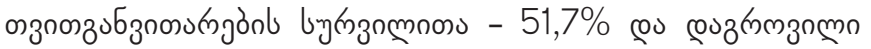

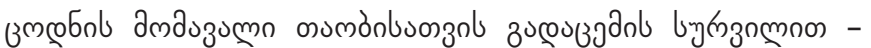
$50,8 \%$

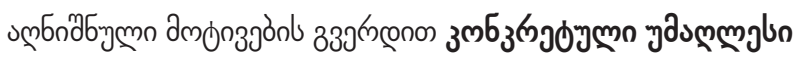

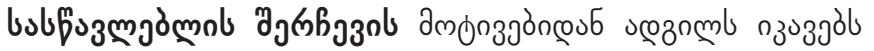

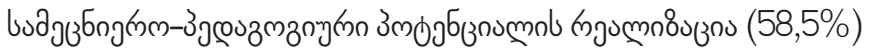

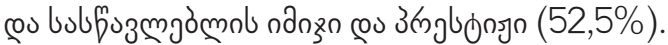

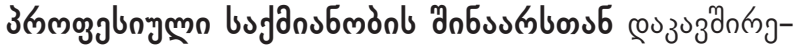

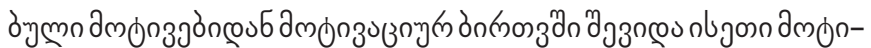

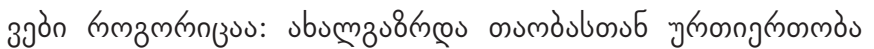

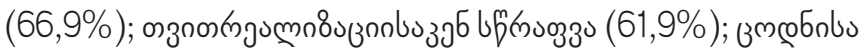

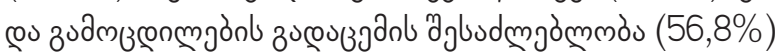

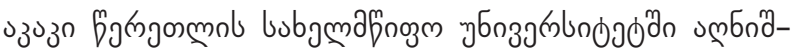

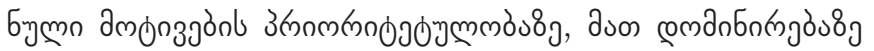

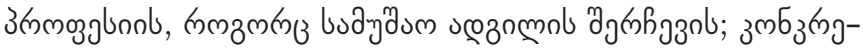

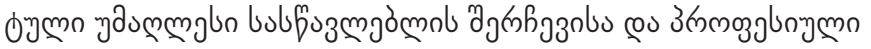

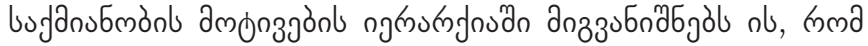

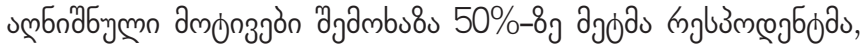

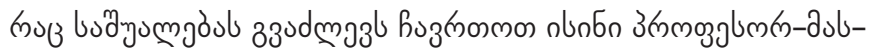

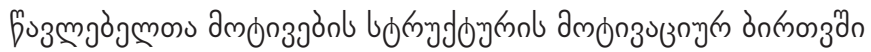
(ob. conog muas).

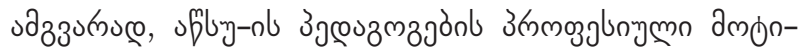

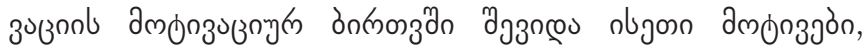

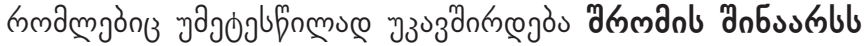

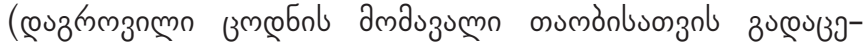

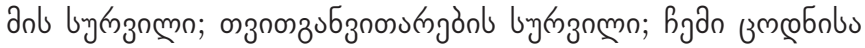

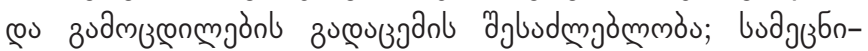

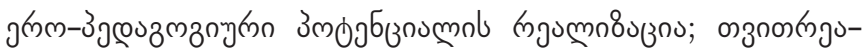

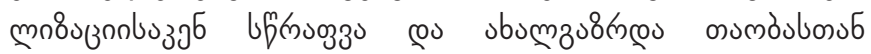

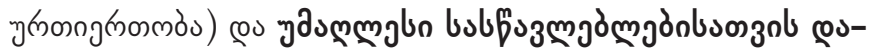

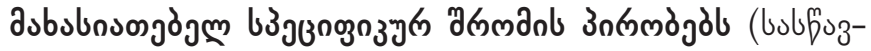

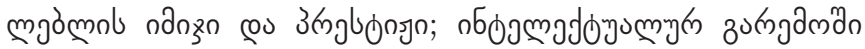

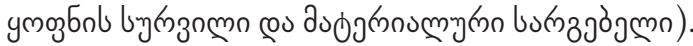

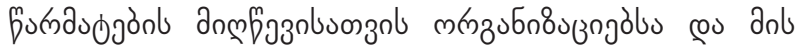

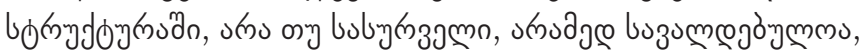

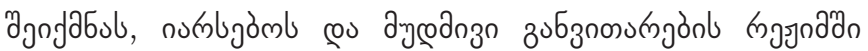

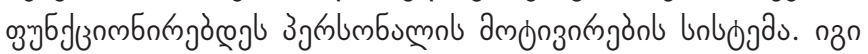

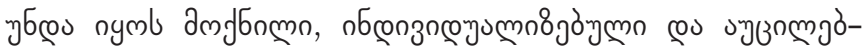

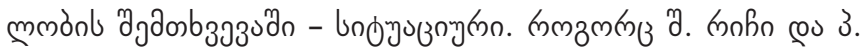

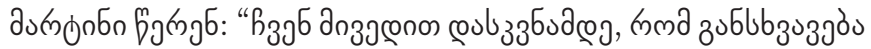

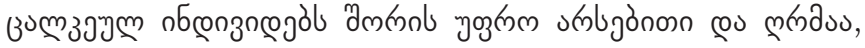

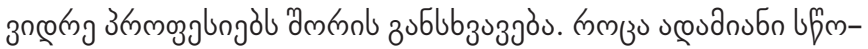

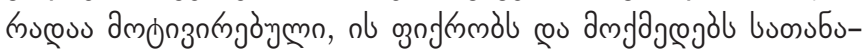

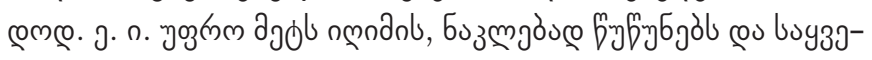

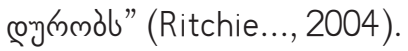

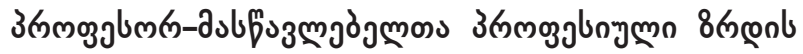

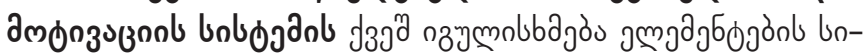

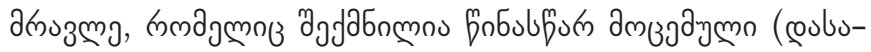

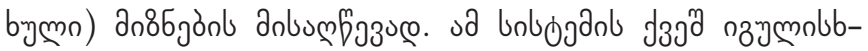

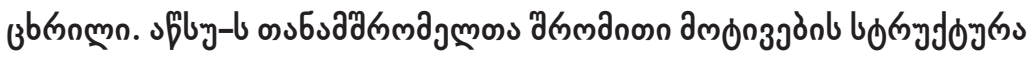

\begin{tabular}{|c|c|c|}
\hline 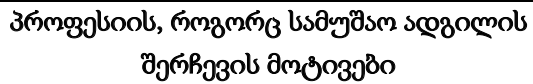 & 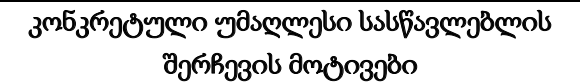 & 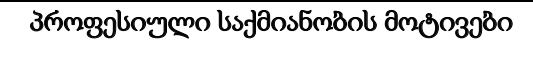 \\
\hline 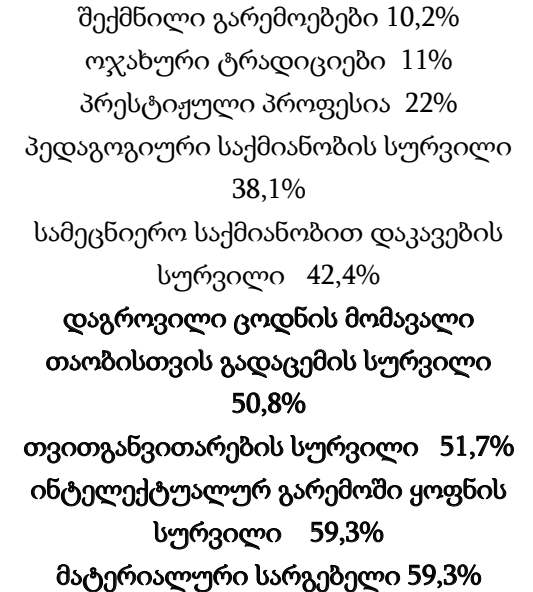 & 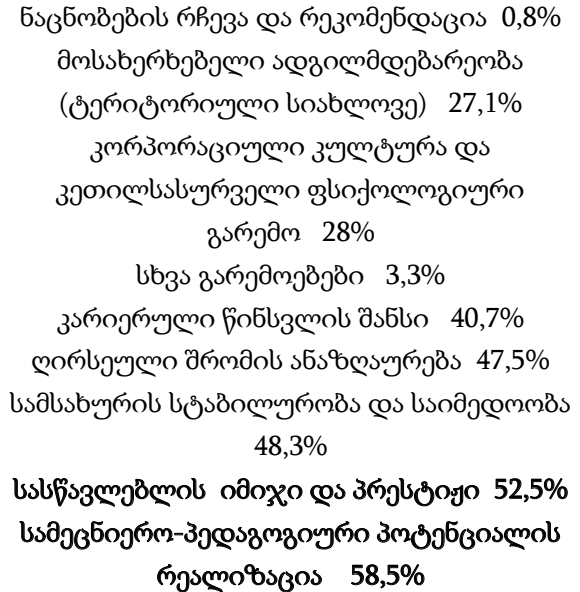 & 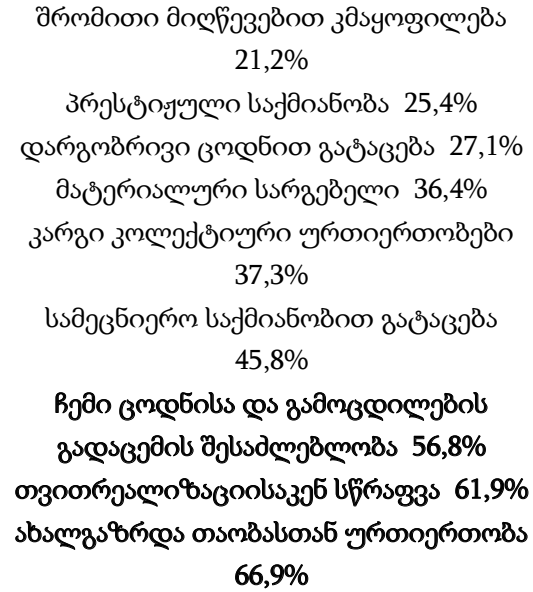 \\
\hline
\end{tabular}




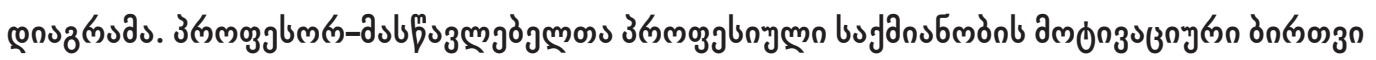

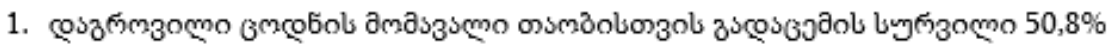

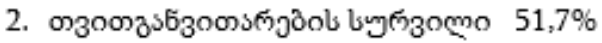

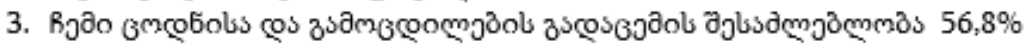

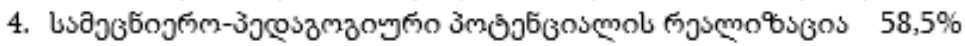

5. Фзоом

6. sbsmаз

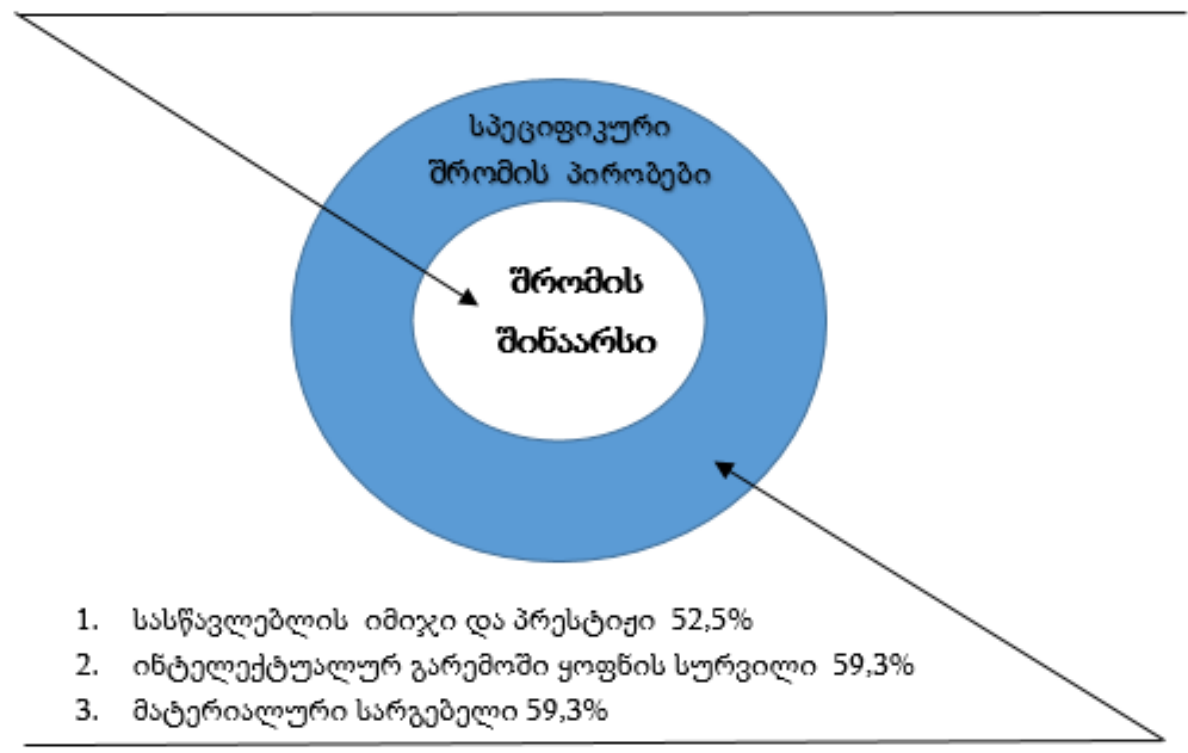

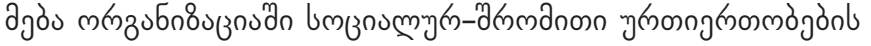

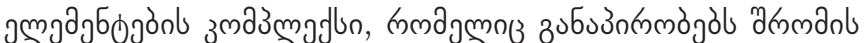

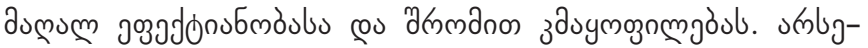
ambl zkmanl amonzuznol bnbojanl amozumn zumnuбon.

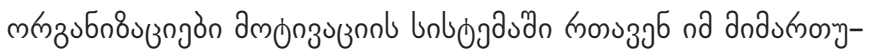

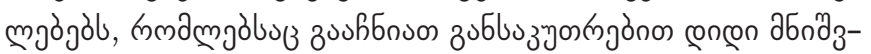

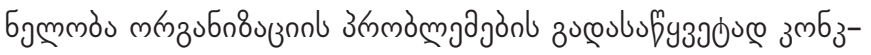

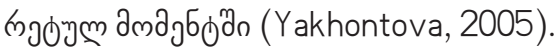

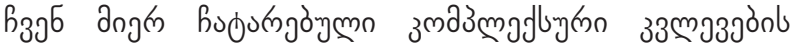

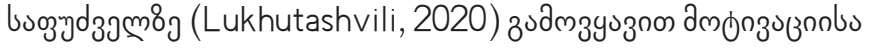

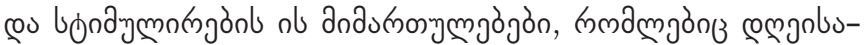

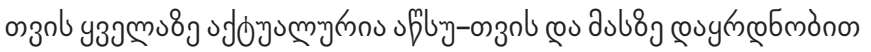

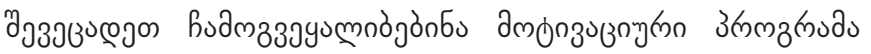

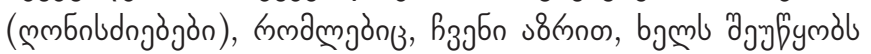
चб

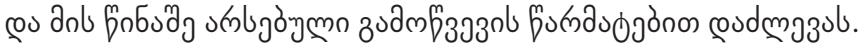

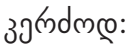

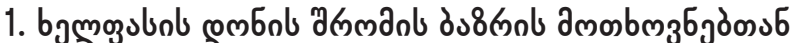

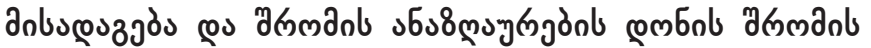

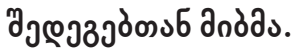

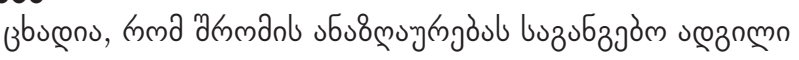

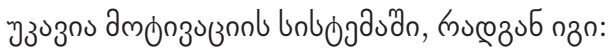

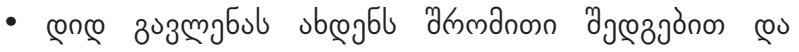

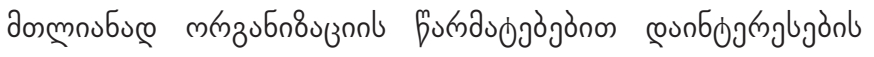
boznomb8?;

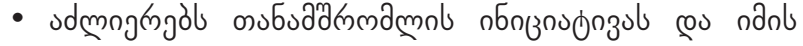

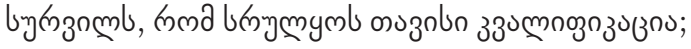

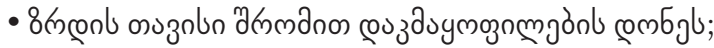

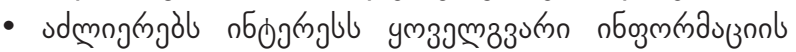

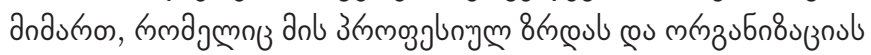
jbajo;

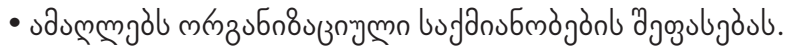

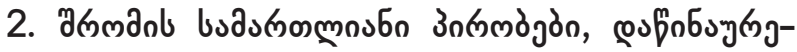

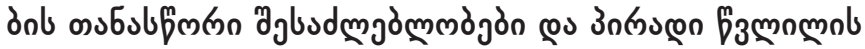

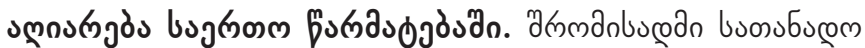

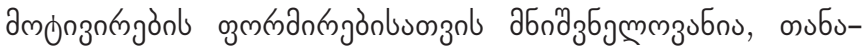

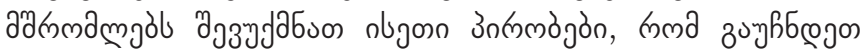

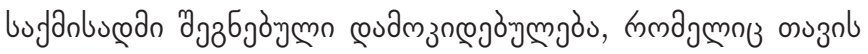

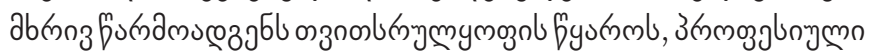

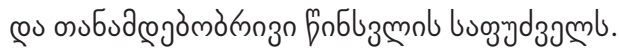

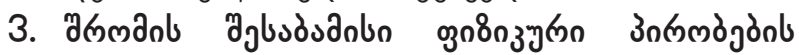

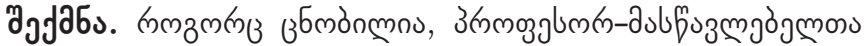

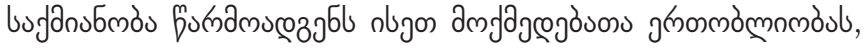

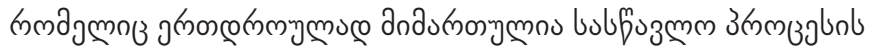

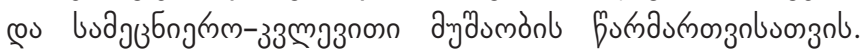

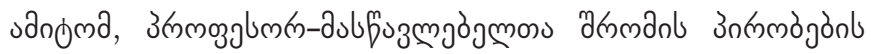

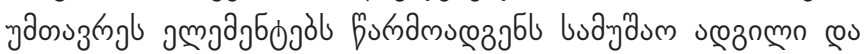

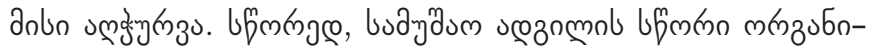

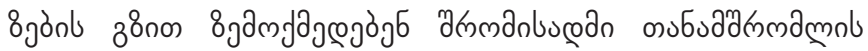

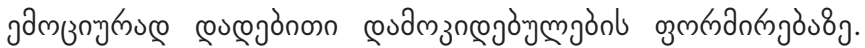




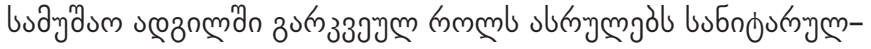

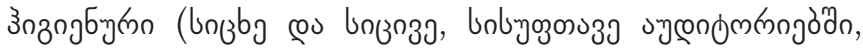

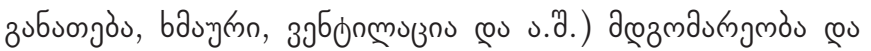

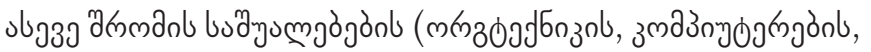

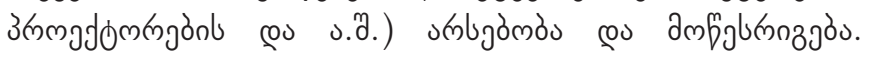

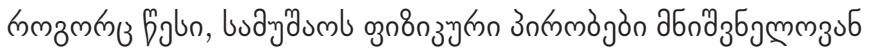

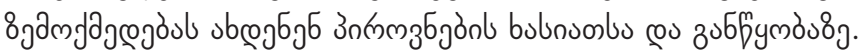

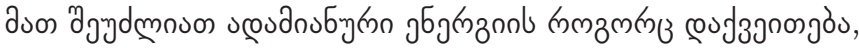
obg odumm gò

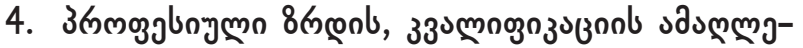

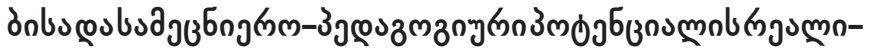

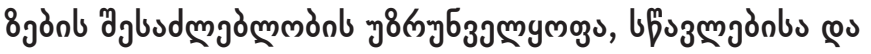

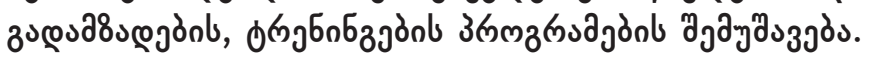

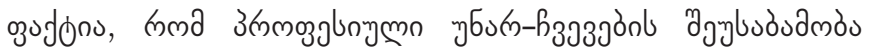

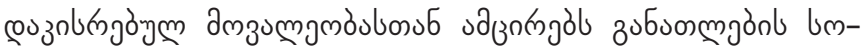

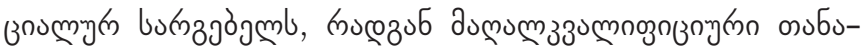

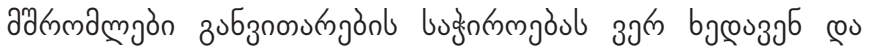

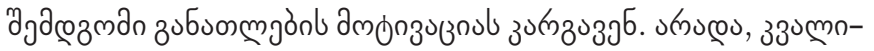

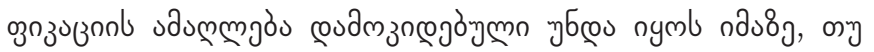

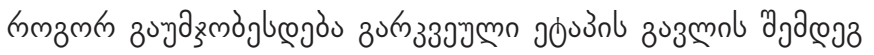

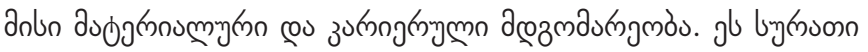

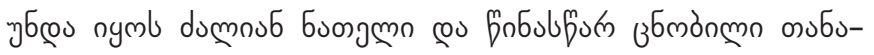

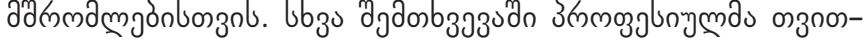

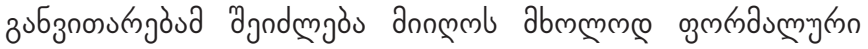

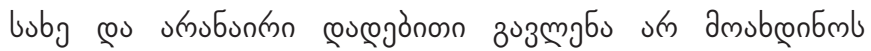

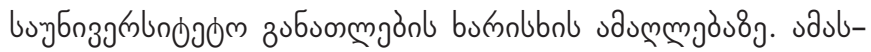

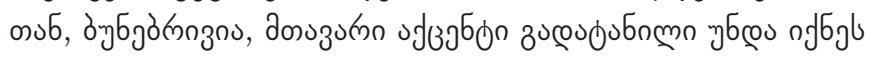

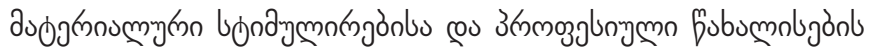
oुगdommgion.

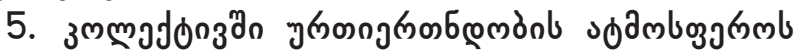

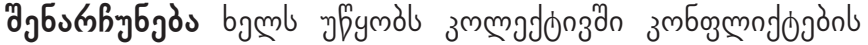

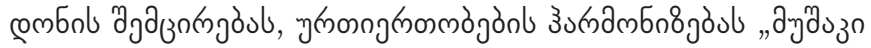

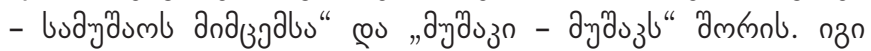

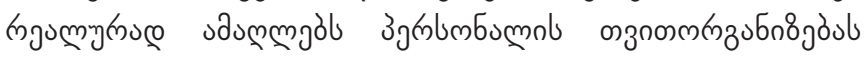

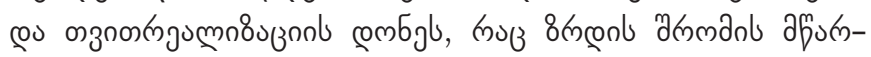

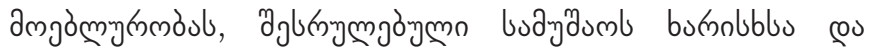
ع ৫nб

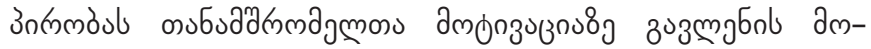

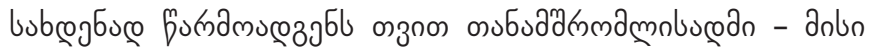

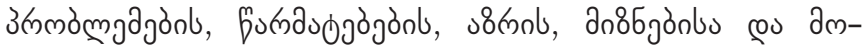

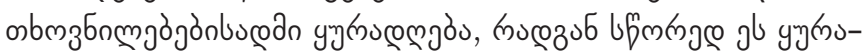

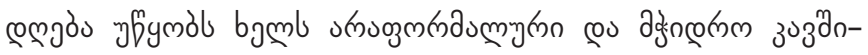

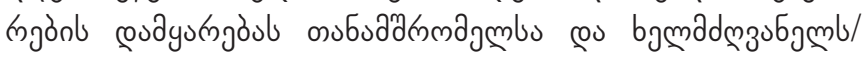
mкrzubnosoznol dmmol.

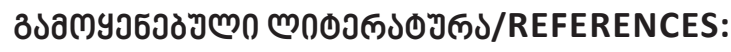

Edward L. D., Richard M. R., (2014). The Importance of Universal Psychological Needs for Understanding Motivation in the Workplace. The Oxford handbook of work engagement, motivation, and self-determination theory. Oxford University Press, 2014:13-33.

Giguashvili G., Makasarashvili T., (2020) Main Challenges of State Policy of Higher Education in Georgia. Globalization and Business, \#9:163-170. (In Georgian).

Gvajaia L., (2017). Assessment of the workforce's activities is an important function of staff management. Globalization and Business, \#4:105-108.

Ilyin, E. P., (2002). Motivation and Motives. Publisher: Piter:270-272. (in Russian).

Kerestesova, M., (2009). The Motivational Program as an Important and Essential Part of Each Organization in the Battle for the Rarest Capital - The Human Potential. Human Resources Management \& Ergonomics, Vol. 3. https://frcatel.fri.uniza. sk/hrme/files/2009/2009_1_05.pdf

Kruglova L.I., From staff motivation to high results// Guide for budgetary organization - 2010- N 8:70 - 76. (in Russian).

Lukhutashvili, N., Valishvili, t., Denosashvili. M., (2020). Modern Methods of Motivation Management in the InnovationOriented Organizations. Austrian Journal of Humanities and Social Sciences Scientific journal, № 3-4, 2020 (MarchApril), Vienna:20-29. DOI:10.29013/AJH-20-3.4-20-29

Machado, M.L., Meira Soares, V., Brites, R., Brites Ferreira, J., Gouveia, O., (2011). A look to Academics Job Satisfaction and Motivation in Portuguese Higher Education Institutions. Procedia- social and Behavioral Sciences 29:1715-1724. https://www.researchgate.net/publication/262450908_A_Look_to_Academics_Job_Satisfaction_and_Motivation_in_ Portuguese_Higher_Education_Institutions

Odishvili K., Nikvashvili M., (2019). Main Challenges of Human Resources Management In Employee Career Development. Globalization And Business, \#7:182-186. (In English)

Ritchie S., Martin P., (2004). Motivation Management: Textbook. manual for universities. Series "Foreign textbook". M: UNITY-DANA:18-26. (in Russian, Tr. from English, ed. prof. E.A. Klimov.)

Yakhontova, E. S., (2005). Effective Technologies of personnel management. Publisher: Piter:36. (in Russian). 